\title{
Response to: Nutritional strategies for the rehabilitation of COVID-19 patients
}

\author{
${\text { Luigia Brugliera } \mathbb{B}^{1} \cdot \text { Alfio Spina }}^{2} \cdot$ Alessandra Giordani $^{1} \cdot$ Sandro lannaccone ${ }^{1}$
}

Received: 1 October 2020 / Revised: 10 October 2020 / Accepted: 26 October 2020 / Published online: 9 November 2020

(c) Springer Nature Limited 2020

\section{To the Editor:}

We read with interest the comments to our previous article by Azzolino et al. about the nutritional management and the swallowing function in SARS-CoV-2 patients [1,2]. These comments highlighted that malnutrition and all its related issues represent a pivotal point, which has not been entirely studied both in COVID-19 and normal patients [1].

Our preliminary study was aimed at formulating an ad hoc protocol for the nutritional management of COVID19 patients during hospitalization in a rehabilitation department [2]. Certainly, the use of validated tools and the multidisciplinary approach was important however, the detection of weight variation of each admitted patient has represented the cornerstone of the management of this particular subtype of patients [2].

The body weight represents the sum of several compartments of the body and it can be influenced by different factors, which cannot entirely explain their composition. In our experience, it is more important the weight variation previously to the beginning of the disease [2]. According to the literature, it is sufficient a variation of $5 \%$ from the last month to diagnose malnutrition. Knowing this weight difference, through a direct interview or telephone contact with relatives, was decisive for setting up an adequate nutritional protocol in our study [2].

Luigia Brugliera

brugliera.luigia@hsr.it

1 Department of Rehabilitation and Functional Recovery, I.R.C.C.S. San Raffaele Scientific Institute, Vita-Salute University, Milan, Italy

2 Department of Neurosurgery and Gamma Knife Radiosurgery, I.R.C.C.S. San Raffaele Scientific Institute, Vita-Salute University, Milan, Italy
According to our data, $46 \%$ of patients showed a weight loss from 5 to $10 \%$ and, $14.8 \%$ higher than $10 \%$, concerning the bodyweight before the COVID-19 disease.

As pointes out by Azzolino et al., the limits of the BMI evaluation can be exceeded by validated tools for body composition analysis [1]. Because it was not possible the use of Dual-Energy X-ray Absorptiometry (DXA), our cohort underwent Body Impendence Assessment (BIA) allowing to evaluate different parameters, such as the Appendicular skeletal muscle mass and index (ASMM and ASMI), which are necessary for sarcopenia diagnosis.

As suggested, the reduction of these two parameters is already enough to diagnose sarcopenia [3]. According to these parameters in our cohort $53.7 \%$ of patients had confirmed sarcopenia, $36.8 \%$ has probable sarcopenia and, only $10.5 \%$ did not show sarcopenia.

Regarding the swallowing deficit, it was reported in more than $90 \%$ of our patients, caused by several factors, such as the generalized sarcopenia, as pointed out by Azzolina et al., which can be responsible of sarcopenic dysphagia [1, 2, 4, 5]. This concept has been already introduced in 2012; however, it is neither easy nor widely accepted the modality of its diagnosis, which has influenced our study [6].

Furthermore, Frajkova et al. have recently published a review of the possible causes of post-intubation dysphagia in COVID-19 patients, pointing out that these patients are at risk for the respiratory symptoms and the decreased pulmonary function [7].

According to these preliminary results, we are widening our study towards those factors able to influence both the clinical course and the outcome of the patient suffering from COVID-19.

Several studies on which factors affect the COVID-19 patients' outcome after hospital discharge are still in progress, such as sarcopenia, the type of previous respiratory 
support during the acute phase of the COVID-19 disease, the cognitive status, and dysphagia.

After hospital discharge, the continuous monitoring of the nutritional status and the possible therapeutic-nutritional intervention is fundamental to ensure a gradual recovery of the optimal bodyweight.

We know that the research on sarcopenia and dysphagia is constantly improving especially in COVID-19 patients. These comments and the future studies are focusing the importance of the nutritional management and the swallowing function in these patients, to improve the functional status and the quality of life.

\section{Compliance with ethical standards}

Conflict of interest The authors declare that they have no conflict of interest.

Publisher's note Springer Nature remains neutral with regard to jurisdictional claims in published maps and institutional affiliations.

\section{References}

1. Azzolino D, Passarelli PC, D'Addona A, Cesari M. Nutritional strategies for the rehabilitation of COVID-19 patients. https://doi.org/ 10.1038/s41430-020-00795-0.

2. Brugliera L, Spina A, Castellazzi P, Cimino P, Arcuri P, Negro A, et al. Nutritional management of COVID-19 patients in a rehabilitation unit. Eur J Clin Nutr. 2020;74:860-3.

3. Cruz-Jentoft AJ, Bahat G, Bauer J, Boirie Y, Bruyère O, Cederholm T, et al. Sarcopenia: revised European consensus on definition and diagnosis. Age Ageing. 2019;48:16-31.

4. Brugliera L, Spina A, Castellazzi P, Cimino P, Arcuri P, Deriu MG, et al. Rehabilitative of COVID-19 patients with acute lower extremity Ischemia and amputation. J Rehabil Med. 2020;52:jrm00094.

5. Brugliera L, Spina A, Castellazzi P, Cimino P, Tettamanti A, Houdayer E, et al. Rehabilitation of COVID-19 patients. J Rehabil Med. 2020;52:jrm00046.

6. Fujishima I, Fujiu-Kurachi M, Arai H, Hyodo M, Kagaya H, Maeda $\mathrm{K}$, et al. Sarcopenia and dysphagia: position paper by four professional organizations. Geriatr Gerontol Int. 2019;19:91-7.

7. Frajkova Z, Tedla M, Tedlova E, Suchankova M, Geneid A. Postintubation dysphagia during COVID-19 outbreak-contemporary review. Dysphagia. 2020;35:549-57. 